\title{
Mark Wallinger zum Thema „Religion im öffentlichen Raum"
}

\author{
Johannes Stückelberger
}

\section{Zusammenfassung}

Viele zeitgenössische Künstler setzen sich mit dem Thema "Religion im öffentlichen Raum" auseinander. Das Phänomen, dass die im Zuge der Säkularisierung unsichtbar gewordene Religion im Zeitalter der Postsäkularisierung eine neue Sichtbarkeit erhält und in den öffentlichen Raum zurückkehrt, geht an ihnen nicht spurlos vorbei. Das wird an vier Arbeiten des englischen Künstlers Mark Wallinger diskutiert. Wo ortet er Religion im öffentlichen Raum? Welche Aspekte von Religion macht er sichtbar? Wie thematisiert er diese Aspekte?

Am Trafalgar Square in London stand von Juli 1999 bis Februar 2000 auf der sogenannten "Fourth Plinth", einem ursprünglich für ein Reiterdenkmal bestimmten, sieben Meter hohen Sockel, ein Mensch in Lebensgrösse, bekleidet nur mit einem Lendentuch, die Hände auf den Rücken gebunden, auf dem Kopf eine goldene Krone aus Stacheldraht. Unverkennbar Jesus, in der Szene, wie er - worauf ein Schild mit dem Titel "Ecce Homo" hinwies - von Pilatus dem Volk vorgeführt wird mit den Worten: "Seht, ein Mensch". Geschaffen hat das Werk Mark Wallinger, der inzwischen zu den bekanntesten englischen Künstlern zählt. ${ }^{1}$ Eine irritierende und gleichzeitig faszinierende Arbeit, angesichts deren sich die Frage stellt: Wie kommt ein zeitgenössischer Künstler auf die Idee, am belebtesten Platz in London eine Jesusstatue aufzustellen?

Wallingers "Ecce Homo" ist kein Einzelfall. Viele Künstler setzen sich heute mit dem Thema "Religion im öffentlichen Raum" auseinander. ${ }^{2}$ Das Phänomen, dass die im Zuge der Säkularisierung unsichtbar gewordene Religion im Zeitalter der Postsäkularisierung eine neue Sichtbarkeit erhält und in den öffentlichen Raum zurückkehrt, geht an der zeitgenössischen Kunst nicht spurlos vorbei. Das sei hier - stellvertretend - an vier Arbeiten Wallingers diskutiert. ${ }^{3}$ Drei Fragen werden uns dabei begleiten: 1 . Wo ortet der Künstler Religion im öffentlichen Raum? 2. Welche Aspekte von Religion macht er sichtbar? 3. Wie thematisiert er diese Aspekte? Wallinger ist ein politischer Künstler, der im und mit dem öffentlichen Raum arbeitet. Religion interessiert inn als Teil dieses öffentlichen Raumes, als öffentliche Sache, die den Alltag, die Politik, unser Denken und Handeln mitbestimmt. Als westlicher Künstler beschäftigt er sich vor allem mit der christlichen Religion. Das Thema Multireligiosität taucht in seinem Werk nur am Rande auf. Dabei erstaunt es immer wieder, mit welcher Selbstverständlichkeit er sich mit religiösen Fragen auseinandersetzt. Und vielleicht noch erstaunlicher ist, wie der Kunstbetrieb ihm darin

\footnotetext{
12001 vertrat Wallinger England an der Biennale in Venedig. 2007 erhielt er den renommierten Turner Prize. An neuerer Literatur über den Künstler sei genannt: Martin Herbert: Mark Wallinger, London 2011; Mark Wallinger: Ausstellungskatalog Braunschweig (Kunstverein)/Aarau (Aargauer Kunsthaus) 2007/2008, Zürich 2007. Zur Rolle der Religion in seinem Werk: Herbert 2011, 98-149; Madeleine Schuppli: Wallinger und die Religion, in: Wallinger 2007, 49-63.

2 Vgl. Johannes Stückelberger: Religion im öffentlichen Raum als Thema der Gegenwartskunst, in: Kunst und Kirche 4 (2008), 5-11 [Heft zum Thema „Religion im öffentlichen Raum, hg. von Johannes Stückelberger].

3 Wallinger hat sich in zahlreichen weiteren Arbeiten mit Religion auseinandergesetzt. Genannt seien "Passport Control“ (1988), "Heaven“ (1988), "Autopsy" (1996), "Seeing is Believing" (1997), "Credo" (2000), "The Word in the Desert" (2000), "Forever an Ever" (2002), "Via Dolorosa" (2002), "The Underworld" (2004), "A ist für Alles" (2005), "The End" (2006), u. a.
} 


\section{Thema: Sichtbare Religion im öffentlichen Raum}

folgt. Auch das kann als Indiz für die neue Öffentlichkeit von Religion gewertet werden, dass die künstlerische Beschäftigung mit ihr kein Tabu mehr ist.

\section{Ecce Homo}

Auf die Frage, was ihn zu der Arbeit "Ecce Homo" veranlasst habe, antwortete der Künstler: „Plötzlich kam mir in den Sinn: Es sind 2000 Jahre nach der Geburt Jesu Christi, damit müsste man sich doch beschäftigen. Aber ich hatte das Gefühl, dass alle so zögerlich waren. Schliesslich leben wir ja in einer multikulturellen Gesellschaft. Das empfand ich ziemlich bizarr, so etwa wie: ,Jesus Christus erwähnen verboten'. Ich machte also etwas, was für mich absolut auf der Hand lag, aber anscheinend niemand zu tun wagte. Ausserdem wurde die Arbeit auch vom Ort selbst angeregt: der Trafalgar Square als einer der meist frequentierten Plätze Londons, ein Ort der Massen, Aufmarschplatz für Feiern und Demonstrationen. Dahin gehört Christus, der dem Mob vorgeführt wird. ${ }^{\prime 4}$ Nun, man könnte Wallinger entgegenhalten, im öffentlichen Raum gebe es eigentlich genug Erinnerungsorte, die auf die unsere Kultur bestimmende christliche Tradition hinweisen, wie etwa, gleich angrenzend an den Trafalgar Square, die Kirche St. Martin in the Fields oder die National Gallery mit ihren reichen Schätzen an christlicher Kunst. Das jedoch genügt dem Künstler offenbar nicht. Er weist uns darauf hin, dass die Religionen, wollen sie lebendig bleiben, ihre Sichtbarkeit immer wieder erneuern müssen.

Im Falle des "Ecce Homo" wird diese Verlebendigung mit Hilfe des Elements der Irritation erreicht. ${ }^{5}$ Neben der 51 Meter hohen Säule in der Mitte des Platzes, dem Denkmal für Admiral Nelson, der in der Seeschlacht von Trafalgar die englische Flotte befehligte, ist Wallingers Jesus kaum sichtbar. Und wohl gerade deshalb war er in der Zeit, da er hier stand, ein Hauptanziehungspunkt. Man fragte sich: Was sucht dieser Mensch auf dem für ihn viel zu großen Sockel? Ist es ein lebender Mensch, in einer Performance als Straßenkünstler? Ein Selbstmordkandidat? Dagegen sprachen seine ruhige Haltung und die geschlossenen Augen. Wer einigermassen mit christlicher Ikonographie vertraut war, erkannte in dem Menschen Jesus. Irritierend war einzig, dass dieser anstelle einer Dorneneine Stacheldrahtkrone trug. Jesus als politischer Häftling? Schnell wurde klar: An diesem öffentlichen Ort der Demonstration politischer, kirchlicher und kultureller Macht ist dieses Kunstwerk ein Gegendenkmal, das daran erinnert, dass Jesus, die zentrale Figur des Christentums, kein Held war, kein politischer Machthaber, kein kirchlicher Würdenträger, keine kulturelle Grösse, sondern ein Mensch, Opfer einer politischen Intrige, wie es sie seither tausendfach gab. Doch warum diese Gelassenheit angesichts seiner Opferrolle? Will uns der Künstler darauf aufmerksam machen, dass den Religionen eine andere Sichtbarkeit zusteht als sie die Politik, die Konsumwelt, der Sport etc. für sich in Anspruch nehmen? Religion ist nicht nur vita activa, sondern auch vita passiva. Religion hat mit Demut zu tun angesichts einer anderen Wirklichkeit, die sich der Sichtbarkeit entzieht. Religion

4 „Im Anfang war das Wort ...”. Mark Wallinger im Gespräch mit Johannes Rauchenberger und Alois Kölbl, in: Kunst und Kirche, 2 (2002), 97-101, das Zitat 100.

5 Zu der Arbeit ausführlicher: Johannes Stückelberger: Zeitgenössische Kunst und religiöse Erfahrung, in: Wilhelm Gräb / Jérôme Cottin (Hg.): Imagination der inneren Welt. Theologische, psychologische und ästhetische Reflexionen zur spirituellen Dimension der Kunst, Frankfurt am Main 2012, 51-66; Sibylla Egli: Christus im öffentlichen Raum - Mark Wallingers „Ecce Homo“, in: Kunst und Kirche, 4 (2008), 12-14; Mark Wallinger: British Pavilion. The $49^{\text {th }}$ Venice Biennale 2001, London 2001; Mark Wallinger: Ecce Homo, Ausstellungskatalog Wien (Sezession) 2000. 


\section{Thema: Sichtbare Religion im öffentlichen Raum}

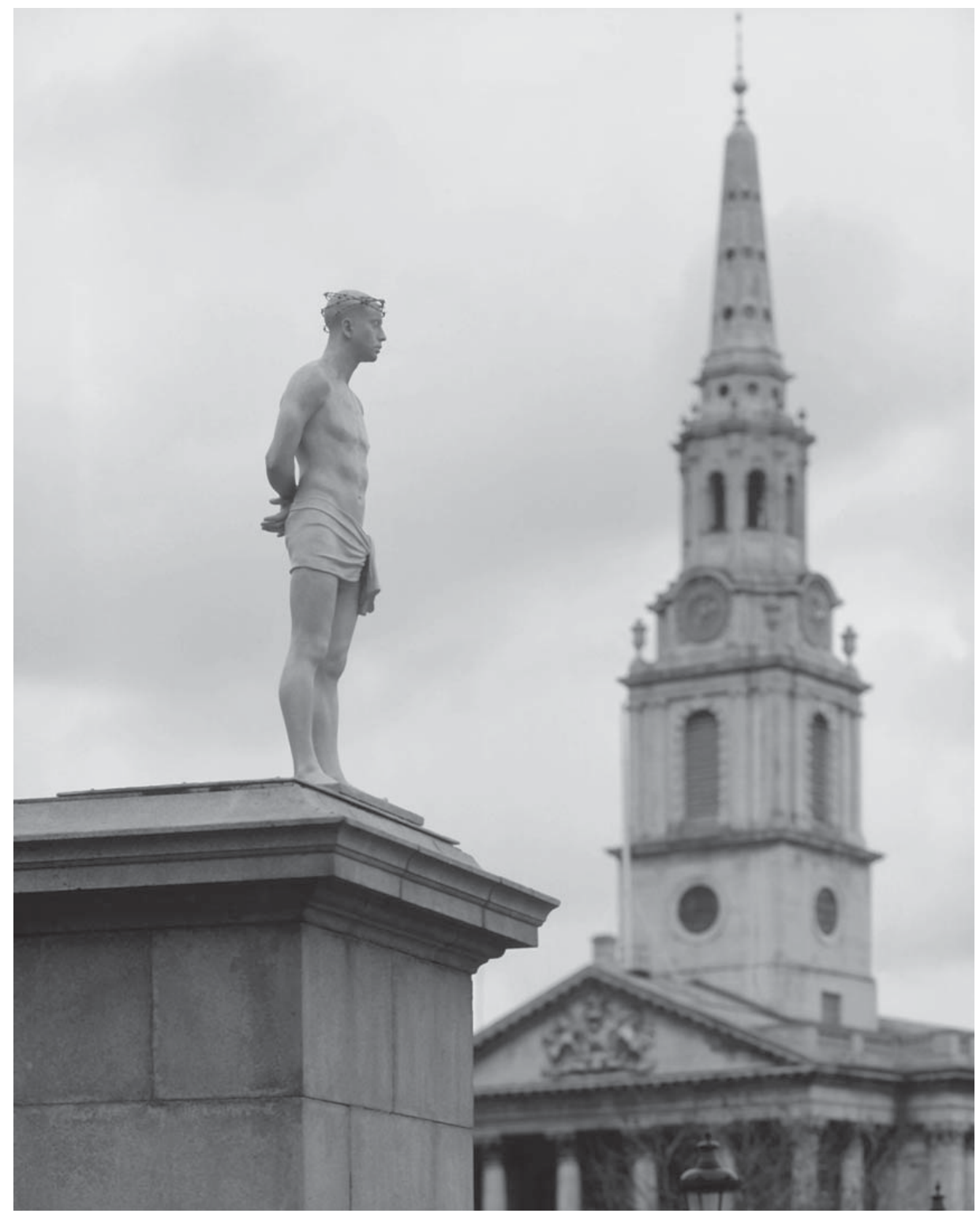

Mark Wallinger: Ecce Homo, 1999, Trafalgar Square London 1999/2000.

Foto: John Riddy. Copyright of the artist. Courtesy Anthony Reynolds Gallery, London.

hat mit Glauben zu tun, mit Standhaftigkeit, mit dem Menschen in seiner Menschlichkeit. Nicht "Seht, ein Held“ lautet der Titel des Werks, sondern "Seht, ein Mensch".

\section{Angel}

Wallinger stellt nicht nur an hochfrequentierten Orten aus, sondern produziert zum Teil seine Werke auch an solchen Orten, so die beiden Videoarbeiten "Angel" und "Threshold to the Kingdom". Angel entstand 1997 in der gleichnamigen Londoner U-Bahnstation, am Fusse einer langen, dreispurigen Rolltreppe. Der siebeneinhalb Minuten dauernde Loop zeigt den Künstler, wie er verkehrt zur Fahrtrichtung auf der untersten Stufe der mittleren Treppe auf der Stelle tritt. Mit einer dunklen Brille, in der Hand einen Blindenstab, rezitiert er immer wieder die gleichen Worte, die ersten fünf Verse des Johannesevangeliums, die in der deutschen Übersetzung lauten: „Im Anfang war das Wort, und 


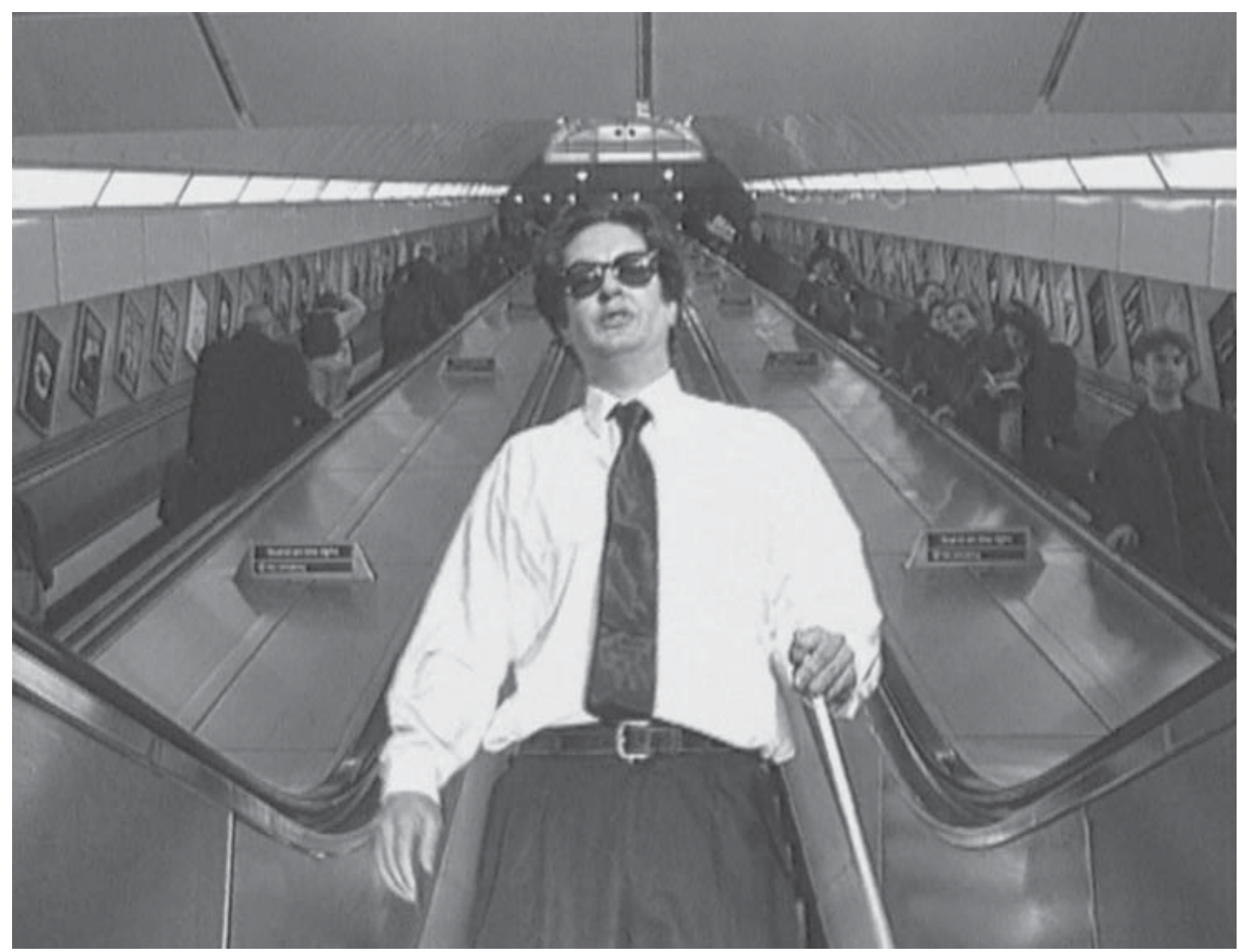

Mark Wallinger: Angel, 1997, Videoprojektion. Foto: Fred Dott. Copyright of the artist. Courtesy Anthony Reynolds Gallery, London.

das Wort war bei Gott, und das Wort war Gott. Im Anfang war es bei Gott. Alles ist durch das Wort geworden, und ohne das Wort wurde nichts, was geworden ist. In ihm war das Leben, und das Leben war das Licht der Menschen. Und das Licht leuchtet in der Finsternis, und die Finsternis hat es nicht erfasst. ${ }^{\prime 6}$ Das Video endet damit, dass der Künstler aufhört zu sprechen und zu gehen, worauf er - zur Musik von Händels "Zadok the Priest" - rückwärts die Treppe hochfährt und oben verschwindet.

An einem Ort, der seinen Namen einem Himmelsboten verdankt, tritt der Künstler mit einer alten Botschaft auf, in der Rolle des "Blinden Glaubens", wie er sie selber bezeichnet. ${ }^{7}$ Auf seine Motive angesprochen, sagt er: „Einmal ist da die Befragung meiner eigenen Sehnsucht, der Hoffnung, des Verlangens nach etwas Transzendentem, das wir alle in uns tragen. Zum andern ist es ein Nachdenken darüber, dass, obwohl wir in einer säkularisierten Gesellschaft leben, die christliche Tradition und ihre Symbolik noch immer tief in jedem von uns eingeprägt sind und uns bestimmen. Ich fand es interessant, das einmal näher beim Namen zu nennen. ${ }^{8}$ Doch nicht nur dass unser Leben und die Gesell-

6 Joh 1,1-5.

7 Die Rolle des „Blinden Glaubens" spielt Wallinger in drei Videos („Angel“, "Hymn“ und "Prometheus“), die er unter dem Titel "Talking in Tongues" zu einer Trilogie zusammenfasste. Zu dieser Trilogie und zur Rolle des "Blinden Glaubens" vgl. Wallinger in: „Das Oberste zuunterst, das Hinterste zuvorderst und verkehrt herum”. Theodora Vischer im Gespräch mit Mark Wallinger, in: Mark Wallinger: Lost Horizon, Ausstellungskatalog Basel (Museum für Gegenwartskunst), 1999, 6-33, speziell 23-31.

8 "Im Anfang war das Wort ..." 2002, 99. 
schaft von Religion geprägt sind, interessiert den Künstler, sondern auch, wie sie es sind. Für das Verständnis der Arbeit ist entscheidend, dass der Film rückwärts abgespielt wird, was man leicht daran erkennt, dass die Personen auf den seitlichen Rolltreppen in die verkehrte Richtung fahren. Gleichzeitig hört man die Worte aus dem Johannes-Prolog richtig, wenn auch etwas undeutlich. Demnach muss der Künstler diese rückwärts gesprochen haben, was in der Tat zutrifft.

Unzählige Assoziationen und Bilder stellen sich ein, die man mit religiösen und im engeren Sinn christlichen Fragestellungen in Verbindung bringen kann: Bilder des Jüngsten Gerichts mit den Erlösten, die auf der einen Seite in den Himmel aufsteigen, während auf der anderen Seite die Verdammten in die Hölle hinabfahren. Oder das Bild der Jakobsleiter, an deren Ende Gott sitzt, und auf der Engel hinauf- und hinabsteigen. Bilder vom Ende der Welt, gleichzeitig erzählt der Johannes-Prolog von deren Anfang, indem er daran erinnert, dass Gott die Welt durch das Wort erschaffen hat. Anfang und Ende, Schöpfung und Vollendung werden zueinander in Beziehung gesetzt und gespiegelt. Der Film schaut vom Ende her auf das Leben, er betrachtet es rückwärts. Und erst aus dieser Perspektive vermag man die Worte des "Blinden Glaubens“, die im normalen Leben unverständlich bleiben, zu verstehen.

Die Arbeit ist hochkomplex, und auch meine Interpretation ist nur ein tastender Versuch, sie auszuloten. Aber immerhin glaube ich so viel verstanden zu haben, dass Wallinger uns sagen will: Wo wir mit unseren Fragen nach Anfang und Ende der Welt sowie nach dem Sinn des Lebens an Grenzen kommen, sind wir auf die Religionen und deren Boten verwiesen, die uns vielleicht keine auf Anhieb verständlichen Antworten geben, aber doch Antworten für unseren Glauben, und die uns vor allem die Dinge aus einer anderen Perspektive sehen lassen.

\section{Threshold to the Kingdom}

Das Video "Threshold to the Kingdom" vom Jahr 2000 drehte Wallinger in der Ankunftshalle des Londoner City Flughafens, also wieder an einem stark frequentierten Ort. Wir sehen eine Türe, die sich in unregelmässigen Abständen automatisch öffnet und durch die die Flugreisenden in die Ankunftshalle kommen: einzeln, in Gruppen, zielstrebig, zögernd, nach Angehörigen Ausschau haltend, einen Moment lang orientierungslos. Die Bewegungen der Ankommenden sind verlangsamt, was ihnen etwas Majestätisches und Schwebendes verleiht. ${ }^{9}$ Dazu ertönt das "Miserere" von Gregorio Allegri: „Herr sei mir Sünder gnädig."

Religiöse Konnotationen stellen sich bei der Arbeit bereits beim Titel ein mit der Doppeldeutigkeit von "Kingdom“. Im Londoner Flughafen ist damit das United Kingdom, das Vereinigte Königreich von Großbritannien und Nordirland, gemeint. Zusammen mit der Musik, die den Film untermalt, assoziiert man mit dem Begriff gleichzeitig das Himmelreich. Der Künstler vergleicht in einem Interview die "Rite de passage“ der Zollkontrolle in den Flughäfen mit der Beichte und Absolution in der Kirche. ${ }^{10}$ Die Türe, durch die die Flugreisenden auf britisches Hoheitsgebiet gelangen, wird in der Arbeit zu einer Metapher für die Schwelle zum "Kommenden Reich“. Interessant ist dabei, dass wir die Ankommenden aus der Perspektive derjenigen sehen, die sich bereits im Himmel befinden.

9 Aus anderthalb Stunden Filmmaterial verwendete der Künstler zweieinhalb Minuten, die er auf siebeneinhalb Minuten dehnte.

10 „Im Anfang war das Wort ...” 2002, 101. 

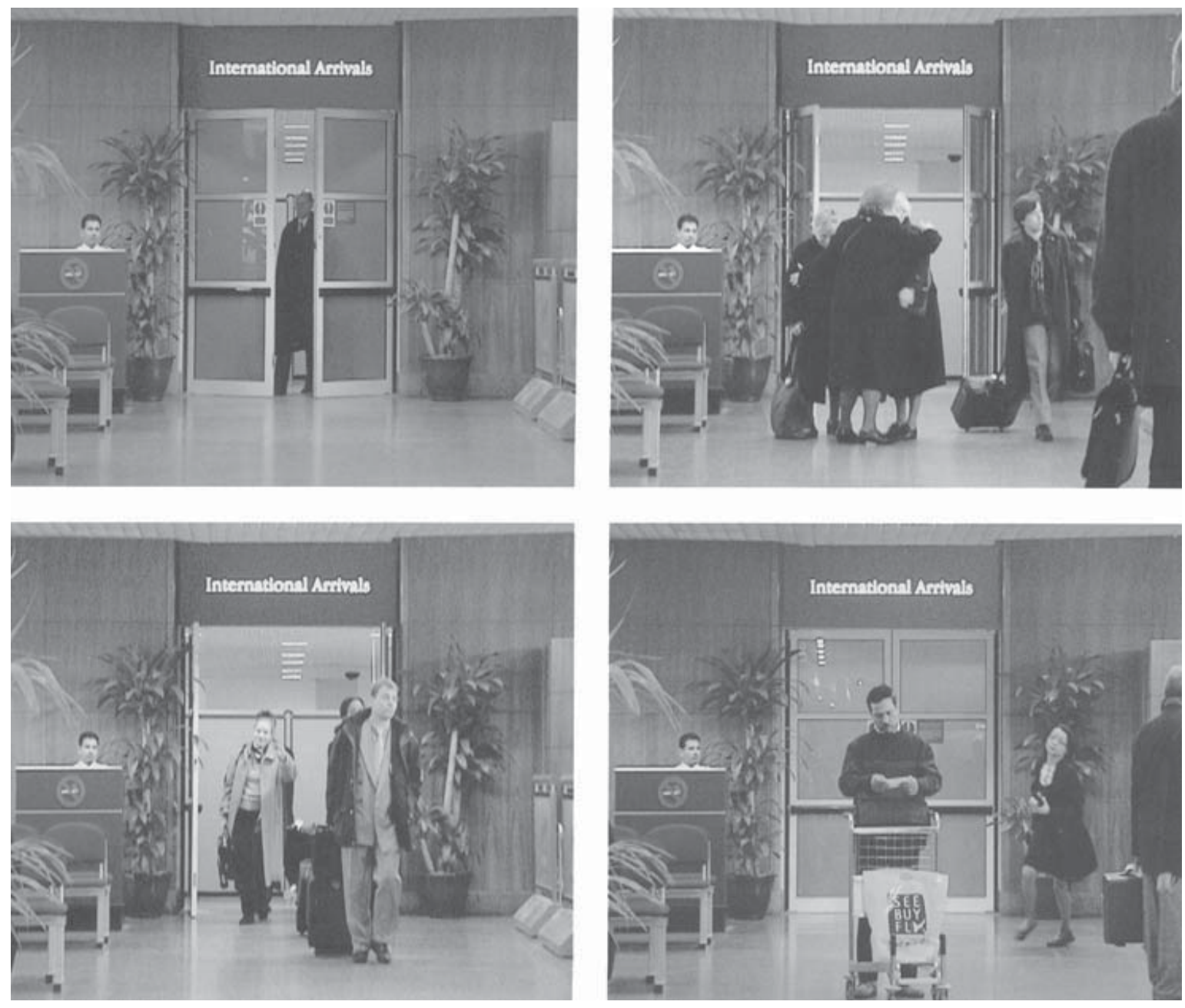

Mark Wallinger: Threshold to the Kingdom, 2000, Videoprojektion. Copyright of the artist. Courtesy Anthony Reynolds Gallery, London.

Auch in dieser Arbeit spielt Wallinger auf zentrale Themen der christlichen Religion an, insbesondere auf die Vorstellungen von Diesseits, Jenseits und Transzendenz. Es stellen sich Fragen wie: Gibt es überhaupt ein Jenseits, und wenn ja, was erwartet uns dort? Wie verhalten sich Diesseits und Jenseits zueinander? Gibt es, wie im Flughafen, auf der Schwelle vom Hier zum Dort eine Grenzkontrolle? Wenn ja, wer sind die Zöllner? Falls wir nicht so sicher sind, im Himmel Aufnahme zu finden, an wen richten wir unsere Bitte um Gnade? Können wir mit dieser Gnade rechnen? Es sind hochtheologische Fragen, mit denen uns die Arbeit konfrontiert. Nicht zuletzt mit diesen: Lässt sich vielleicht gar nicht unterscheiden zwischen Diesseits und Jenseits? Was meint die Rede vom Himmel auf Erden? Warum wählt Wallinger als Metapher für den göttlichen Himmel nicht jenen Himmel, aus dem die Reisenden kommen, sondern die Ankunftshalle, den sicheren Boden des Flughafens? Und warum interpretiert er den Übertritt ins Jenseits, in eine andere Wirklichkeit, nicht als Abschied, sondern als Ankommen?

\section{Zone}

2007 wurde Wallinger zur Teilnahme an der Ausstellung "Skulptur Projekte Münster 07" eingeladen. Wie schon bei den Vorgängerausstellungen war den Eingeladenen die Auf- 
Mark Wallinger: Zone, 2007. Installationsansicht Skulptur Projekte Münster 07. Copyright of the artist. Courtesy Anthony Reynolds Gallery, London.

gabe gestellt, eine Arbeit zu realisieren im öffentlichen Raum von Münster, mit einem inhaltlichen Bezug zur Stadt. Wallinger spannte um die Altstadt, auf einem Kreis von fünf Kilometern Länge und in einer Mindesthöhe von viereinhalb Metern, eine Angelschnur, von Haus zu Haus und Baum zu Baum. Obwohl kaum sichtbar, war diese "Skulptur" eine der meistbeachteten der Ausstellung. ${ }^{11}$

In "Zone“, so der Titel der Arbeit, geht es um Grenzen und Grenzziehungen, um Eingrenzung und Ausgrenzung: auch dies - wenn auch nicht nur - ein religiöses Thema. Der Künstler markiert die Grenzen nicht mit einer Mauer, sondern mit einer dünnen, kaum sichtbaren Schnur. Will er damit auf die subtilen Grenzen in unseren Städten hinweisen? Will er sagen, dass die Konflikte der Religionen nicht nur auf deren Sichtbarkeit im öffentlichen Raum zurückzuführen sind, sondern auch mit unsichtbaren Differenzen und Abgrenzungen zu tun haben? Zu der Arbeit angeregt haben Wallinger ein kurz zuvor im Nordwesten von London errichteter Eruv und die Diskussionen, die dieser auslöste. Der Eruv ist in der jüdischen Tradition ein exakt eingegrenztes Gebiet, in dem einige der Verbote, die orthodoxe Juden am Sabbat einhalten müssen, entfallen. Mit dem Eruv wird der öffentliche Raum zum gemeinschaftlichen Haus erklärt, er ist somit das Gegenteil des Ghettos.

11 Die folgenden Informationen stammen aus einem Text, den Wallinger für den Katalog der Ausstellung verfasste: Mark Wallinger: Zone (Zone), in: Brigitte Franzen / Kasper König / Carina Plath (Hg.): Skulptur Projekte Münster 07, Ausstellungskatalog Münster, Köln 2007, 257-263. 


\section{Thema: Sichtbare Religion im öffentlichen Raum}

In den in London geführten Diskussionen ging es beispielsweise um die Frage, ob das Gebiet des Eruv weiterhin anderen Nutzungen zur Verfügung stehe. Dass eine Kulturgruppe oder Religionsgemeinschaft im öffentlichen Raum Grenzen zieht und Zonen schafft, bedeutet für Wallinger nicht, dass Angehörige anderer Gruppierungen aus diesen Zonen ausgegrenzt sind. Der öffentliche Raum erlaubt seiner Meinung nach Mehrfachbelegungen. Im Zusammenhang mit der Arbeit "Zone“ spricht er denn auch nicht von Grenzen, sondern von Türen und Schwellen, die er in die Luft gezeichnet habe. ${ }^{12}$ Das Thema sind hier demnach nicht die Grenzen, die die Religionen vermeintlich trennen, sondern jene Grenzen, die sie miteinander verbinden: die Grenzen zwischen dem Diesseits und dem Jenseits, Grenzen, die für die Religionen nicht Mauern sind, sondern Orte des Übergangs, Türen und Schwellen. Die Kunst - so Wallinger - hat ihren Platz an solchen Orten des Übergangs. ${ }^{13}$

\section{Schluss}

Was sagt Wallinger zum Thema "Religion im öffentlichen Raum“? Ein kurzes Fazit.

1. Der öffentliche Raum ist von den Religionen geprägt. Religion als öffentliche Angelegenheit, die die ganze Gesellschaft betrifft, gehört nicht in den privaten, sondern in den öffentlichen Raum. Sie bedarf einer neuen Sichtbarkeit.

2. Religion im öffentlichen Raum vermittelt Werte. Sie stellt uns im Alltag Fragen wie: Woher kommen wir, wer sind wir, wohin gehen wir? Sie appelliert an unsere Menschlichkeit. Sie konfrontiert uns mit Perspektivenwechseln. Sie verkehrt Oben und Unten, Stark und Schwach, Groß und Klein. Sie setzt uns in Beziehung zueinander und zu Gott. Sie öffnet Türen und begleitet uns über Schwellen.

3. Das Thema "Religion im öffentlichen Raum“ muss weder missionarisch noch ironisch diskutiert werden, man kann es sachlich und ernsthaft angehen.

4. Viele zeitgenössische Künstler setzen sich mit dem Thema "Religion im öffentlichen Raum" auseinander. Die Zeit der privaten Mythologien, der Beschäftigung mit Religion ausschliesslich als einer Privatsache, ist vorbei. Religion interessiert heute unter dem Aspekt ihrer Öffentlichkeit.

5. Die Kirchen können in der zeitgenössischen Kunst eine Bestätigung für die in ihren eigenen Reihen wachsende Erkenntnis finden, dass Religion eine öffentliche Angelegenheit ist, die Sichtbarkeit verlangt.

12 A.a.0., 258.

13 Mark Wallinger: The Russian Linesman. Frontiers, Borders and Thresholds, Ausstellungskatalog London (The Hayward) u. a., London 2009, 62. 